Meta

Journal des tradlucteurs

Translators' Journal

\title{
Proceeding(s) : procédure(s) ou non?
}

\section{Raynald Boult et Bernard Méchin}

Volume 28, numéro 2, juin 1983

URI : https://id.erudit.org/iderudit/002124ar

DOI : https://doi.org/10.7202/002124ar

Aller au sommaire du numéro

Éditeur(s)

Les Presses de l'Université de Montréal

\section{ISSN}

0026-0452 (imprimé)

1492-1421 (numérique)

Découvrir la revue

Citer cet article

Boult, R. \& Méchin, B. (1983). Proceeding(s) : procédure(s) ou non? Meta, 28(2), 172-178. https://doi.org/10.7202/002124ar d'utilisation que vous pouvez consulter en ligne.

https://apropos.erudit.org/fr/usagers/politique-dutilisation/ 


\section{PROCEEDING(S) : PROCÉDURE(S) ou NON?}

Les difficultés que suscite la traduction de «proceeding(s)» tiennent à la polysémie du mot. Ces difficultés s'aggravent quand en français on doit choisir un terme qui est lui-même polysémique, par exemple procédure.

Or, comme le dit Becquart (les Mots à sens multiples dans le droit français), la présence d'un mot polysémique dans un texte de loi l'expose à être détourné de sa signification première, le terme en question venant à être pris dans une autre acception que celle envisagée par le législateur.

Les observations qui suivent ne portent que sur les acceptions les plus courantes. Il n'est pas exclu que d'autres puissent se présenter dans d'autres contextes. Les termes proposés pour rendre les divers sens de «proceeding(s)» le sont donc sous la réserve ci-dessus.

\section{Nota}

S'il est certain que pour certaines acceptions ( 4 et 5 par exemple) «proceeding(s)» doit être au pluriel, il ne semble pas que le nombre conditionne rigoureusement le sens. Ainsi, le mot, employé comme entrée, est au singulier dans Black, alors qu'il est au pluriel dans Webster. Le sens indiqué est pourtant le même.

* Source du présent article : Étude exhaustive sur «Proceeding(s)» réalisée par Raynald Boult, ancien bibliothécaire à la Cour suprême du Canada, pour le compte du ministère fédéral de la Justice. 
Les deux nombres sont donc employés indistinctement, selon les textes, sans incidences sémantiques. Aussi serait-il périlleux, semble-t-il, de faire du nombre de «proceeding» une norme pour la traduction.

On peut néanmoins constater une tendance à l'emploi du pluriel en anglais, et au contraire une nette préférence pour le singulier en français, le mot "procédure " ayant déjà en soi un sens générique et par conséquent pluriel.

\section{ACCEPTIONS}

1) Opérations faites par une entité dans l'exercice de ses attributions

Dans cette acception, «proceedings" s'entend en un sens large qui n'est pas propre à la langue juridique.

Oxford: "2. The carrying on of an action or series of actions; action, course of action ; conduct, behaviour..."

Webster: "...Act, deed, a particular thing done."

a) Présence de cette acception dans les textes:

- "The Board may make rules governing its proceedings under the Act." Subsection 3(5) Agricultural Products Act, A-5.

- "The Corporation may... make by-laws for the regulation of its proceedings and generally for the conduct and management of its activities." Sec. 13, Canadian Film Development Corporation Act, C-8.

- "The Minister shall annually lay before Parliament a report of all proceedings under this Act for the preceding fiscal year." Sec. 9, Trans-Canada Highway Act, T-12.

b) Équivalences françaises relevées dans cette acception :

- «Une loi organique fixe la composition de la Haute Cour, les règles de son fonctionnement... » Art. 67, Constitution de la République française.

- Organisation et fonctionnement de l'Assemblée, Règlement et fonctionnement de l'Assemblée Nationale (française) - Titre premier.

- «...de vous rendre compte de l'activité de la Cour de Cassation durant l'année écoulée. » Rapport de la Cour de Cassation, 1975.

c) Termes proposés :

activité

fonctionnement

travaux

2) Action ou poursuite en justice dans toutes ses phases

Black: "The word (proceeding) may be used synonymously with "action" or "suit" to describe the entire course of an action at law or suit in equity."

Oxford: "3; a legal action or process;"

a) Présence de cette acception dans les textes :

_- "The Bank has commenced proceedings..." Sec. 58, Banks and Banking Law Revision Act, 1980, B-1.

- "No statement... is... admissible in evidence in any legal proceeding." Sec. 26 Corrupt Practices Inquiries Act, C-33.

b) Équivalences françaises relevées dans cette acception :

- «...toutes actions contre les associés... Les poursuites de créanciers sociaux...» Dalloz, D. Com. p. 82.

— «...le magistrat fixe les délais nécessaires à la mise en état de la procédure.» Dalloz. 
— «En tout état de la procédure, il peut entendre...» Dalloz.

- Convention Européenne sur la Transmission des Procédures Répressives.

$-\mathrm{v}^{\circ}$ Procédure : «2. Instruction judiciaire d'un procès. » Littré.

- $v^{\circ}$ Procédure : «entamer une procédure de divorce». Grand Larousse.

- «Marche des procédures. Les tableaux suivants dressent l'état des affaires...». Rapport, Cour de Cassation, 1975.

- «Une fois réglé le problème de compétence, il importe que l'instance soit poursuivie...» Dalloz, P.C. p. 445.

— «...la marche des instances...» Dalloz, P.C. p. 452.

- «...action au sens de procédure judiciaire...» Code de commerce, p. 35.

- «La péremption ne concerne que les instances qui relèvent de l'ordre judiciaire; donc elle ne s'applique ni aux instances devant les tribunaux administratifs et les tribunaux des pensions ni aux instances criminelles». J.C.P.C., F. 681.

\title{
c) Observations
}

L'emploi du pluriel procédures est souvent un calque de l'anglais, notamment lorsque le terme «procédure» est employé au sens d'une action engagée contre une personne. Ainsi, l'anglais «to initiate proceedings» doit se rendre par : «engager [introduire, entamer] une procédure». Le pluriel est cependant possible pour marquer la généralité, ainsi que le montrent certains des exemples donnés ci-dessus.

Autre exemple : Les procédures visées par l'article...

d) Termes proposés :

action

poursuite

instance

procédure(s)

\author{
affaire \\ litige \\ opération (judiciaire, juridique) \\ ...en justice
}

3) Une ou plusieurs phases d'une action organisées comme telles

\section{A. Domaine judiciaire}

Black: "Term 'proceeding' may refer not only to a complete remedy but also to a mere procedural step that is part of a larger action or special proceeding."

O.E.D. : "...any step taken in any cause by either party."

a) Présence de cette acception dans les textes:

- Reconciliation proceedings. "8.(1) On a petition for divorce it is the duty of the court..." Sec. 8, Divorce Act, D-8.

b) Équivalences françaises relevées dans cette acception :

- «Les actes d'instruction, d'enquête préliminaire» Dalloz P.P., p. 122.

— «Les procédures devant le président du tribunal...» Dalloz P.C., p. 652.

- «le premier acte de l'instruction du procès...» Dalloz .C., p. 580.

— «...l'annulation des procédures d'instruction...» C.P.P.F., a. 174.

- «Il leur appartient d'accomplir les actes de procédure dans les formes et délais requis.» C.P.C.F., a.2.

- «La nullité des décisions et actes d'exécution relatifs aux mesures d'instruction... » C.P.C.F., a. 175.

- «...les actes de poursuite ou d'instruction... » Dalloz, P.P., p. 129.

- «...l'expression faire acte de procédure signifie agir dans la procédure; ce n'est donc pas le dépôt... mais en général toute activité ayant trait à l'instance...» Académie de Droit International, Recueil des cours, Vol. 65, p. 663.

- «...toutes procédures faites postérieurement à... seront nulles». C.P.C.F. (anc.). a.344. 
c) Termes proposés : acte (de procédure, d'instruction) procédure(s) mesure

diligence(s)

\section{d) Remarques}

(i) Noter le nombre d'exemples où le terme «procédure " est employé au pluriel. (ii) Le mot «diligences» a été introduit dans le nouveau code de procédure civile français avec le sens d' «actes valables». Il est à prévoir que la jurisprudence et la doctrine françaises feront usage de plus en plus du terme, précisément dans une des acceptions de «proceedings». La définition qu'en donne Capitant est la suivante :

«Diligences»: Action de remplir les formalités nécessaires à la conclusion d'une opération juridique. Ex.: faire les diligences pour parvenir à une vente par adjudication, pour obtenir une décision judiciaire.

Voici quelques exemples d'emploi du terme :

«...accordé... le pouvoir d'interrompre la péremption par ses diligences. » Encyclopédie Dalloz (Péremption d'instance).

«...desquels il résulte que cette partie a eu de justes motifs d'arrêter ses diligences...»D.S. 1973 p. 460.

«C'est pourquoi elle a retenu toutes les diligences telles... la constitution d'avoué, la mise au rôle. » D.S. 1973 , p. 461.

«L'instance est périmée lorsqu'aucune des parties n'accomplit de diligence pendant deux ans.» C.P.C.F., a.386 (nouveau).

\section{B. Domaine plus large, juridique ou non}

Random House : "A particular action or course of action." Webster (New Collegiate) : "Procedure."

a) Présence de cette acception dans les textes:

- Proceedings upon reports. "22. The Secretary of State of Canada shall publish the report in the Canada Gazette." Sec. 22, Disfranchising Act, D-7.

_ "...in any step or proceeding precedent to the order...". Sec. 167, Canada Temperance Act, T-5.

b) Équivalences françaises relevées dans cette acception :

— «...les diverses formalités préalables..." Dalloz Adm., p. 100.

- «..la procédure de vérification..." Dalloz Comm., p. 166.

— «Procédures diverses» C.P.C.F. a. 812.

— «Procédure arbitrale: moyen pacifique de régler un conflit.» Basdevant.

- «Sous le terme général de défenses, on groupe tous les procédés qui permettent au défendeur... » Dalloz P.C., p. 58.

— «Sur la nature de ces mesures..., l'art. 81 ne fait aucune restriction... » Normand, p. 346 .

c) Termes proposés

procédure(s)

mesure

procédé (moyen de droit)

acte

formalité(s)

opération

démarche

étape

4) Débats d'un procès, d'une audience

Oxford Companion to Law : $v^{\circ}$ Trial : "The general term for proceedings in a court of first instance."

a) Présence de cette acception dans les textes:

- "At any proceedings before a court martial the accused person has the right..." National Defence Act, N-4, sec. 156. 
b) Équivalences françaises relevées :

— «...si les débats ne sont pas achevés au cours de la première audience.... Dalloz, P.C., p. 625 .

- «...la direction du procès est abandonnée aux plaideurs. " Dalloz, P.C., p. 499.

— «Des audiences du juge du tribunal d'instance...» C.P.C.F. (anc.) a.8.

c) Tèrmes proposés :

débats audience

procès

faits, incidents

5) Délibérations d'une assemblée ou compte rendu des séances d'une assemblée, etc.

Il peut s'agir des seules décisions que prend l'assemblée, ou de tous les incidents d'une séance, ou encore du compte rendu intégral d'un colloque, d'une conférence, d'un symposium, etc.

Collins : "5. the events of an occasion, meeting, etc. ..."

Oxford A.D. :"2. What takes place at a formal meeting of a society, etc."

Oxford: "(in pl.) published report of discussions or conference."

a) Présence de cette acception dans les textes:

- "The Chairman shall cause minutes of the proceedings at the meeting..." Bankruptcy Act, B-3.

- Proceedings of the Sixth International Symposium on Comparative Law.

b) Équivalences françaises relevées dans cette acception :

— «es délibérations prises en ces matières seraient nulles...» Dalloz, Com., p. 186.

— "Les délibérations sont constatées par des procès-verbaux...»Dalloz, Com. p. 198.

— «Conditions de validité des décisions de l'assemblée...» Dalloz, Com., p. 188.

- Actes du deuxième colloque international de linguistique et de traduction, Montréal, 1972, Meta, vol. 18, nos 1-2, 1973.

- «...et le texte de notre communication... à paraître dans les Actes édités par J. Caudmont... » Giessen..., in Meta, vol. 27, ${ }^{\circ}$ 2, juin 1982, p. 197.

c) Termes proposés :

compte rendu, procès verbal décisions

délibérations

travaux

\section{ANNEXE}

Différents sens du mot. «procédure »

Le mot «procédure» est un terme polysémique évoquant une notion protéiforme. Il «désigne des réalités juridiques différentes». (Dalloz-Dictionnaire de droit, Procédures civile et commerciale.)

Il ressort des textes que le terme peut avoir les sens suivants :

1) «Il vise l'ensemble des formalités à suivre pour obtenir d'un tribunal la solution d'une difficulté d'ordre juridique.» (Dalloz). C'est pour ainsi dire une vue statique de la procédure envisagée comme un ensemble de règles. Les codes de procédure consignent ces règles.

Vérification :

- «Elle aura donc pour objet de préciser les règles à observer par les particuliers...» Rouard, p. 838.

— «a procédure est donc l'ensemble des règles à observer devant les tribunaux...» Camuzet, s.1.

- «...elle a pour objet de préciser les règles que les particuliers doivent observer devant les tribunaux...» Solus, p. 13. 
2) Le mot peut aussi signifier la marche, en droit et en fait, des formalités prescrites dans une instance. On pourrait dire que c'est une vue dynamique de la procédure. «On appelle ainsi les actes, significations, expéditions et instructions faits dans le cours d'un procès. » Ferrière (Procédures).

Vérification :

- Le Dictionnaire Robert, au mot procédure, donne, parmi ses exemples : «Engager, intenter, introduire une procédure".

- Grand Larousse de la langue française: Procédure: «...Par ext. Ensemble des actes accomplis en un procès jusqu'au moment où intervient le jugement définitif. Une procédure volumineuse».

- «Le juge chargé de suivre la procédure...». Dalloz.

3) Le terme peut désigner une «série de formalités qui doivent être successivement remplies pour aboutir à un résultat déterminé ». Perraud-Charpentier.

Procédure. «Manière de procéder, d'agir, d'accomplir certains actes, d'exercer une fonction. Le terme procédure peut être entendu dans un sens plus ou moins large.» Basdevant. «Procédure» La forme dans laquelle on doit intenter les demandes en justice, y défendre...» Bugnet-Pothier.

Vérification:

- «la procédure de vérification...» Dalloz D.C. p. 166

— «L procédure d'élaboration.... Dalloz, Adm. p. 99.

- «La procédure du sursis...* Dalloz, Adm. p. 226

- «Les procédures d'expropriation...» C.P.C.Q. a.12.

4) Au sens large c'est la «branche de la science du droit ayant pour objet de déterminer les règles d'organisation judiciaire, de compétence, d'instruction des procès et d'exécution des décisions de justice. * Capitant.

N.B. En certains cas, la reproduction du mot «proceedings» dans le texte français est superfiue.

"The application for a suspension of proceedings..."

"La demande de sursis... " C.P.C.Q., a.603.

"Incidental Proceedings

Incidents" C.P.C.Q., a.199.

De même, sa traduction est parfois inutile en français quand on emploie une locution ou une certaine forme de style.

«En tout état de cause» C.P.C.Q., a. 404

"...to be present at the execution of letters rogatory and to cooperate in these proceedings..."

«....assister à l'exécution des commissions rogatoires et d'y collaborer...»

Conseil de l'Europe - Comité des ministres Rec. R(80)7.

RaYNald Boult et BERNARD MÉchin

Abréviations des ouvrages cités

Basdevant : J. Basdevant, Dictionnaire de la terminologie du droit international

Black: Black's Law Dictionary, 5th ed.

Camuzet: C.E. Camuzet, Procédure civile

Capitant : H. Capitant, Vocabulaire juridique

C.P.C.F.: Code de procédure civile français

C.P.C.Q.: Code de procédure civile du Québec

C.P.P.F. : Code de procédure pénale francais

Dalloz, Adm. : Précis Dalloz, Droit administratif, $9^{\mathrm{e}}$ éd.

Dalloz, Com. : Précis Dalloz, Droit commercial, $10^{\mathrm{e}}$ éd.

Dalloz, P.C. : Précis Dalloz, Procédure civile, $19^{\mathrm{e}}$ éd.

Dalloz, P.P. : Précis Dalloz, Procédure pénale, $11^{\mathrm{e}}$ éd.

Dreuilhe et Deysine: Dictionnaire anglais-français et lexique français-anglais des termes politiques, juridiques et économiques

D.S. : Recueil Dalloz-Sirey

Ferrière: Ferrière, Dictionnaire des termes de droit

J.C.P.C.: Jurisclasseur - Procédure civile

Normand: J. Normand, Le juge et le litige

Oxford (O.E.D.): Oxford English Dictionary

Oxford A.D. : Oxford American Dictionary

Perraud-Charmantier: A. Perraud-Charmantier, Petit dictionnaire de droit

Random House: The Random House Dictionary of English Language

Rouard : P. Rouard, Traité élémentaire de droit judiciaire privé

Solus: Solus et Perrot. Droit judiciaire privé

Webster: Webster's Third International Dictionary 